\title{
X-Ray Eclipse Timing in the LMXB EXO0748-676
}

\author{
Michael T. Wolff*, Paul S. Ray* and Kent S. Wood* \\ ${ }^{*}$ E.O. Hulburt Center for Space Research, Naval Research Laboratory, Washington, DC 20735
}

\begin{abstract}
Orbital period changes are an important diagnostic for understanding low mass X-ray binary (LMXB) accretioninduced angular momentum exchange and overall system evolution. We present our most recent results for the eclipse timing of the LMXB EXO 0748-676. Since its discovery in 1985 it has apparently undergone three distinct orbital period "epochs", each characterized by a different orbital period than the previous epoch. We outline the orbital period behavior for EXO 0748-676 over the past 18 years and discuss the implications of this behavior in light of current theoretical ideas for LMXB evolution.
\end{abstract}

\section{INTRODUCTION}

There are eight currently known low mass X-ray binary (LMXB) systems that undergo full or partial eclipses. Such systems are important for the study of evolution in LMXBs because the eclipse edges provide timing markers that make possible the systematic observation of orbital period changes. The best studied orbit in this group is that of EXO 0748-676 which has been X-ray active since 1985 [1]. This long timeline has allowed an unprecedented look at its orbital dynamics [see 2, and references therein]. The emerging picture for the orbital period behavior, however, is anything but the expected smooth variations in $P_{\text {orb }}$ and $\dot{P}_{\text {orb }}$ based on theoretical calculations done to date [e.g., see 3 , and references therein]. Rather, the observed period changes are discontinuous across multiple distinct epochs, and large apparent changes in $P_{\text {orb }}$ of the order seconds can be observed on timescales as short as one orbit. The magnitude of the observed changes in orbital period are much larger than expected from LMXB evolutionary theory. This is likely an indication that the observed variations in $P_{\text {orb }}$ are short timescale effects of angular momentum redistribution in the system and are masking the underlying long term orbit evolution. The Rossi X-Ray Timing Explorer (RXTE) satellite has allowed us to monitor systematically the orbit of EXO 0748-676 in an effort to delineate and understand the process of angular momentum exchange and its effects on the EXO 0748-676 orbit. In this conference paper we report our recent progress.

\section{RXTE OBSERVATIONS OF EXO 0748-676}

We now have 85 additional RXTE mid-eclipse time measurements over and above those analyzed in Wolff et al. [2] for EXO 0748-676. Wolff et al. reported that the orbit period increased around 1990 in a somewhat discontinuous manner by $\sim 7.9 \mathrm{~ms}$ since its discovery in February 1985. The cumulative data set of mid-eclipse timings considered in that paper ended in December 2000. Our present data set extends to observations done through October 5, 2003. O-C (Observed - Calculated) diagrams for all published mid-eclipse timings of EXO 0748-676 plus the additional timings we present here (a total of 262 mid-eclipse time measurements) are shown in Figures 1 and 2.

The additional eclipse measurements display a remarkable behavior. When the eclipses observed after December 2000 are included in our analysis the orbit period appears to return to a value close to the orbit period observed during the EXOSAT/GINGA epoch. We fit a three-constant-period model $\left(P_{\text {orb } 0}, P_{\text {orb } 1}\right.$, and $\left.P_{\text {orb2 } 2}\right)$ to these data in which we constrain the phase to be constant across the instantaneous period changes but let the cycles $\left(n_{b 0}\right.$ and $\left.n_{b 1}\right)$ of the period change be free parameters (see Table 1). The best-fit model gives a positive period change of about $P_{\text {orb } 1}-P_{\text {orb0 }}=8.19 \pm 0.06 \mathrm{~ms}$ similar to that reported in [2] during the GINGA to early RXTE era. However, the additional data does not continue the trend of random wandering about the new period. Instead, the apparent EXO 0748-676 orbital period undergoes another change near MJD 51710 (near cycle 35136) of $P_{\text {orb2 }}-P_{\text {orb } 1}=-6.75 \pm 0.02 \mathrm{~ms}$. This brings the net change in orbit period in the EXOSAT-RXTE eras (February 1985 through October 2003) to only $P_{\text {orb2 }}-$ 


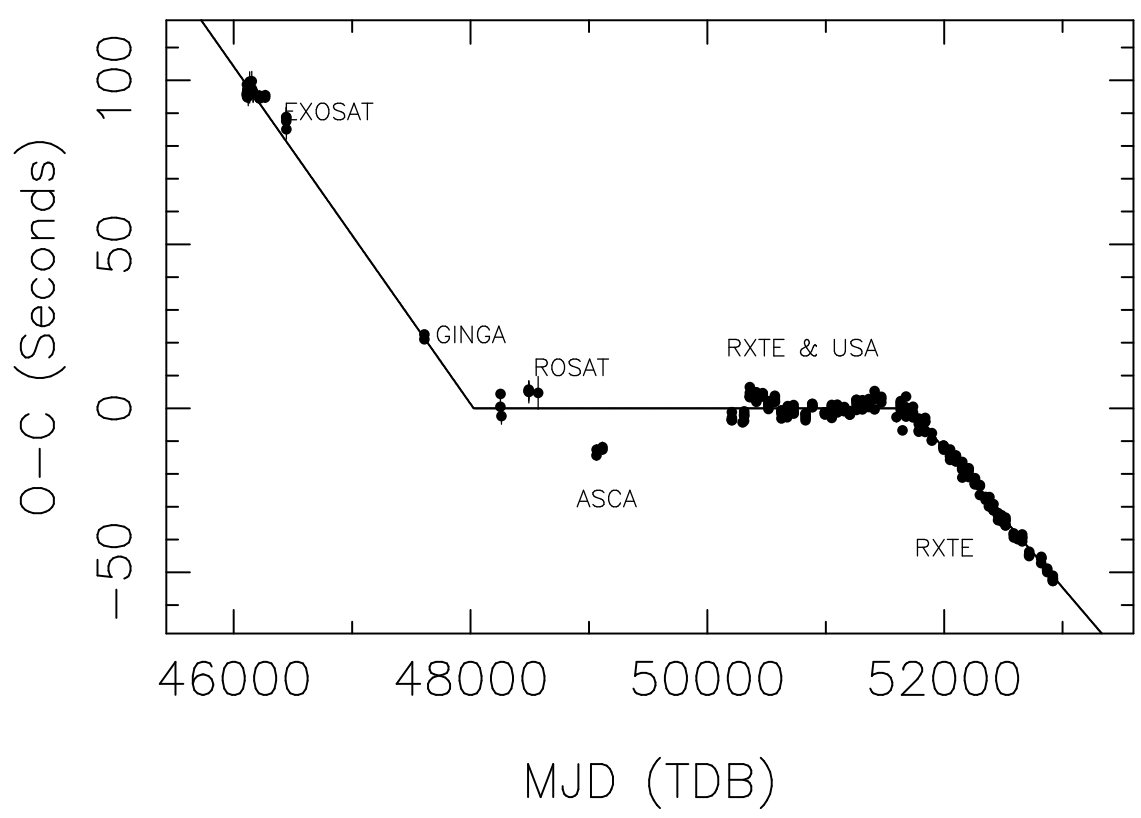

FIGURE 1. The $\mathrm{O}-\mathrm{C}$ residuals for all published mid-eclipse timings of EXO 0748-676 plus those additional timings we present here for a total of 262 mid-eclipse timings. All times are in Modified Julian Days corrected to the solar system barycenter. The measurement errors are as shown and except for a few cases are less than $\sim 1-2$ seconds. The solid line represents the DoubleBroken Constant Period model for these data discussed in the text. The large deflection of the ASCA data away from either solution may be attributable to large excursions due to accumulated jitter as discussed in [2]. The systematic wandering of the orbit period caused by cumulative jitter prior to MJD 51710 is apparent. After this date, however, the orbit period takes on a new value that is only 1.4 ms larger than the apparent orbit period prior to MJD 48026.

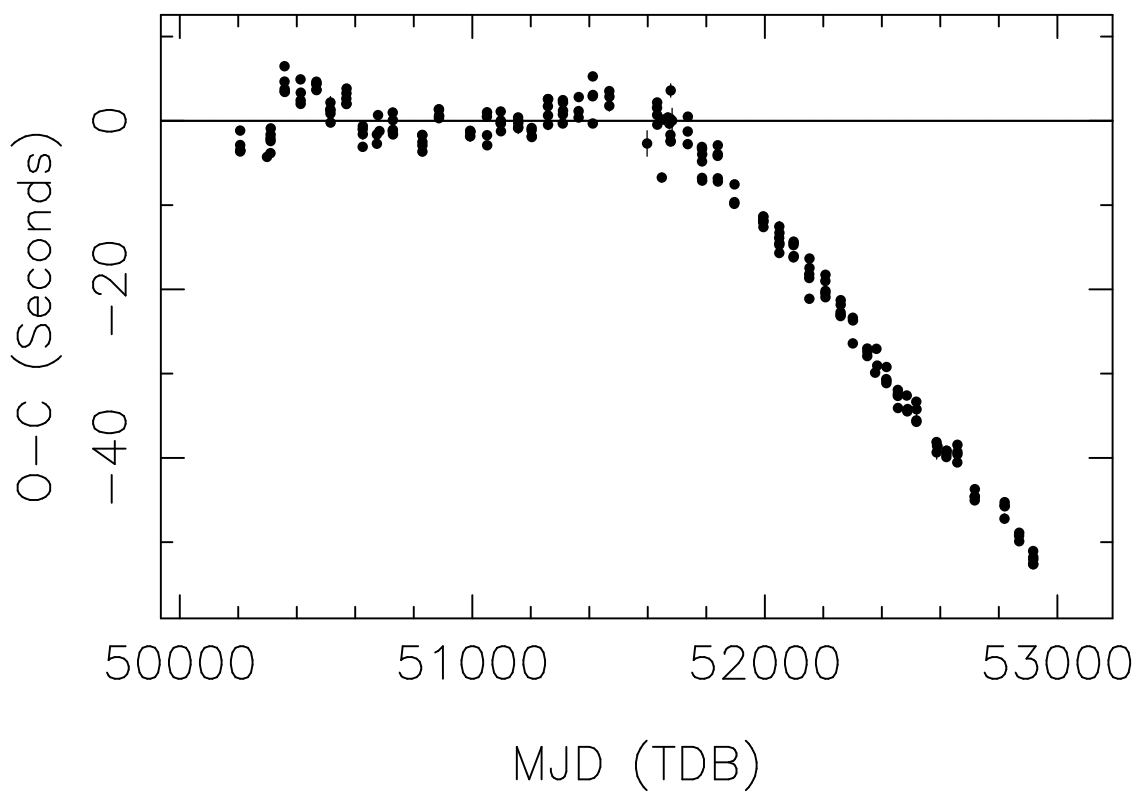

FIGURE 2. The $\mathrm{O}-\mathrm{C}$ residuals for all USA and RXTE mid-eclipse timings of EXO $0748-676$ plotted against the middle period of the Double-Broken Constant Period solution in Table 1. All times are in Modified Julian Days corrected to the solar system barycenter. The solid line represents a constant period solution for the $\mathrm{O}-\mathrm{C}$ residuals and is clearly unacceptable as a model for these data. The systematic wandering of the orbit period caused by cumulative jitter prior to MJD 51710 is apparent. After this date, however, the orbit period takes on a new value of $P_{\text {orb }}=0.15933774511(16) \times 10^{-10}$ (days) and the wandering about the mean period is noticeably reduced. 
$P_{\text {orb0 }}=1.45 \pm 0.06 \mathrm{~ms}$, significantly less than reported in [2]. Note that the orbit period in the present epoch $\left(P_{\text {orb2 }}\right)$ is distinct from the period in the EXOSAT/GINGA epoch $\left(P_{\text {orb0}}\right)$ according to our analysis.

\section{CUMULATIVE JITTER IN X-RAY MID-ECLIPSE TIMINGS}

The $\mathrm{O}-\mathrm{C}$ residuals in Figure 2 appear correlated, that is, they are often systematically grouped above and below the zero residual line. In some cases the positive or negative variations in the mid-eclipse residuals can persist for many months. We have referred to this variation in the apparent orbital period as "jitter", and apparent changes in orbital period can be caused by accumulated intrinsic jitter in a constant orbital period. "Measurement error" is simply the random error associated with our measurement of the individual mid-eclipse times. We take "intrinsic jitter" to mean the systematic wandering of the mid-eclipse timings around a smooth underlying model that can not be accounted for by random uncorrelated measurement errors. In particular, the systematic wandering of the mid-eclipse residuals apparent in Figure 2 can be accounted for by the accumulated intrinsic period jitter in individual orbit cycles. In order to investigate the observational measurement error inherent in our timing analysis and the intrinsic period jitter that is caused by other mechanisms (possibly mechanisms inside the LMXB system) we apply Maximum Likelihood Method [MLM, see 4] to estimate the parameters of a model for the orbital evolution that simultaneously includes an orbit period, an orbit period derivative, non-zero random intrinsic scatter (variance $\sigma_{\varepsilon}^{2}$ ), and non-zero random measurement error (variance $\sigma_{e}^{2}$ ). We give results for EXO 0748-676 in two cases: (a) RXTE and USA data prior to MJD 51710, and, (b) the RXTE data after MJD 51710. A MLM model for the EXO 0748-676 RXTE and USA mid-eclipse timing data prior to MJD 51710 that includes a non-zero period derivative, intrinsic jitter and measurement error is not statistically preferred over a model with only intrinsic jitter and measurement error (a model with no intrinsic period jitter is strongly rejected according to the MLM analysis). The MLM analysis suggests that the intrinsic jitter in the eclipse timings is characterized by $\sigma_{\varepsilon} \sim 0.13 \mathrm{~s}$ with measurement error $\sigma_{e} \sim 1.3 \mathrm{~s}$, similar to the results in [2]. However, for the RXTE data after MJD 51710 the MLM analysis suggests that while the measurement error in the eclipse timings remains roughly constant at $\sigma_{e} \sim 1.2 \mathrm{~s}$, the intrinsic period jitter is greatly reduced to $\sigma_{\varepsilon} \sim 0.028 \mathrm{~s}$. Also, as was the case for the pre-MJD 51710 data, a model for the post-MJD 51710 timing data that includes a non-zero period derivative, intrinsic jitter and measurement error is not statistically preferred over a model with only intrinsic period jitter and measurement error. Whatever process resulted in the abrupt change in the orbital period after MJD 51710 also brought about a reduction in the magnitude of the intrinsic orbital period jitter according to the MLM analysis.

\section{DISCUSSION}

The X-ray eclipse timings for EXO 0748-676 appear to show three distinct orbital periods in three successive epochs along with significant intrinsic period jitter in these epochs. Furthermore, inspection of Figure 2 shows that during the early part of the RXTE epoch (until approximately March 2000) the period jitter is especially prominent. After this date the orbit period changes again by $\sim-6.7 \mathrm{~ms}$ nearly returning to the value characteristic of the EXOSAT-GINGA epoch. Such abruptly changing orbit periods are observed in a number of Algol binaries [see the discussion in [5] and references found therein] although with larger amplitude in $\Delta P_{\text {orb }} / P_{\text {orb }}$. For Algol systems $\Delta P_{\text {orb }} / P_{\text {orb }} \sim 10^{-5}$ whereas in EXO0748-676 we find $\Delta P_{\text {orb }} / P_{\text {orb }} \sim 10^{-6}$. [6] attributed the abrupt period changes in Algol systems to magnetic activity in a convective secondary star inducing changes in its quadrupole moment and altering the orbital angular momentum. If this explanation is applicable to the period changes in EXO 0748-676 [see also 7] then around the March 2000 magnetic activity in the secondary was altered in some manner changing the secondary's influence on the system orbital angular momentum distribution. Before this date the orbit period jittered around a mean value but the jitter is reduced after this date. This may imply that magnetic activity in the secondary also was reduced, perhaps as a result of magnetic cycling similar to the 11-year cycle in the Sun. Small changes in radius of the secondary are predicted by the magnetic activity explanation of Algol orbit period changes put forward by Applegate and Patterson [8] due to the changing magnetic pressure support for the outer layers of the secondary star. The data in Figure 3 suggest that the radius of the occulting star decreased across the MJD 51710 boundary. Totality duration is $\sim 7$ seconds shorter after MJD 51710 than before MJD 51710. However, a spherically symmetric decrease of $1-2 \%$ in the radius of the secondary star is unlikely and other nonspherical changes in the radius of the secondary must be considered as well.

The measurements shown in Figures 1, 2, and 3 give a magnitude and direction for the abrupt orbital period changes and the magnitude and direction of the abrupt change in the duration of X-ray totality. Any attempt to understand these data will have to pose some sort 


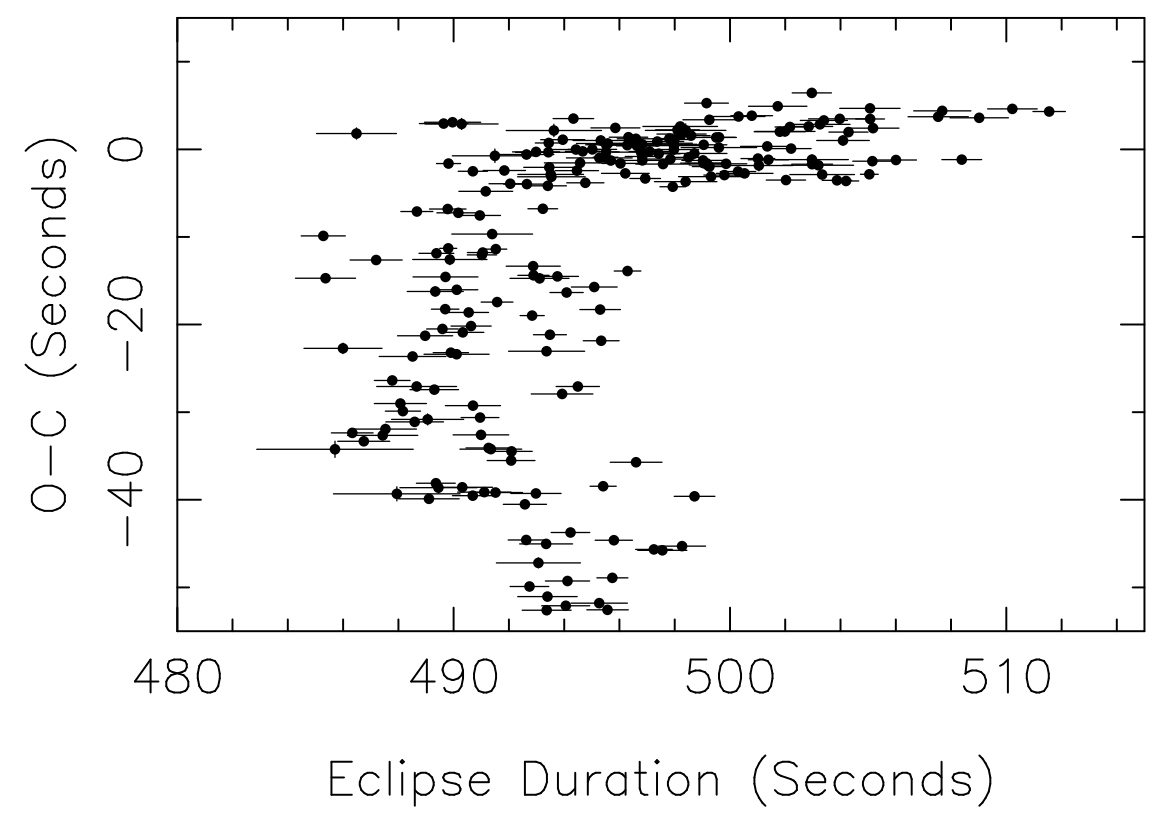

FIGURE 3. The durations of all RXTE observed eclipses plotted as a function of observed $\mathrm{O}-\mathrm{C}$ residual for EXO $0748-676$. The points at increasing negative $\mathrm{O}-\mathrm{C}$ values are the same eclipses observed after the dramatic change in period near MJD 51710 in Figure 2.

of almost instantaneous change in the binary system parameters with an accompanying redistribution of the orbital and spin angular momenta rather than a slow change in the system because of the effects of mass exchange.

\section{ACKNOWLEDGMENTS}

This research is supported by the Office of Naval Research, the NASA Astrophysical Data Program, and the NASA RXTE Guest Observer Program.

\section{REFERENCES}

1. Parmar, A. N., White, N. E., Giommi, P., and Gottwald, M., ApJ, 308, 199 (1986).

2. Wolff, M. T., Hertz, P. L., Wood, K. S., Ray, P. S., and Bandyopadhyay, R. M., ApJ, 575, 384-396 (2002).

3. Podsiadlowski, P., Rappaport, S., and Pfahl, E., ApJ, 565, 1107 (2002).

4. Koen, C., MNRAS, 283, 471 (1996).

5. Simon, V., $A \& A S, \mathbf{1 3 4}, 1-19$ (1999).

6. Hall, D. S., ApJ, 380, L85-L87 (1991).

7. Hertz, P., Wood, K. S., and Cominsky, L. R., ApJ, 486, 1000 (1997).

8. Applegate, J. H., and Patterson, J., ApJ, 322, L99-L102 (1987).

TABLE 1. Orbital Ephemerides of EXO 0748-676

\begin{tabular}{llc}
\hline Parameter & & Value \\
\hline \multicolumn{2}{c}{ All Eclipse Timing Data } \\
\hline$T_{0}(\mathrm{MJD} / \mathrm{TDB})$ & $=$ & $46111.0752010(42)$ \\
$P_{\text {orb0 }}($ day $)$ & $=$ & $0.15933772838(66)$ \\
$n_{b 0}($ cycle $)$ & $=$ & $12019.5(63.8)$ \\
$P_{\text {orb } 1}($ day $)$ & $=$ & $0.15933782322(10)$ \\
$n_{b 1}($ cycle $)$ & $=$ & $35136.4(11.4)$ \\
$P_{\text {orb2 }}($ day $)$ & $=$ & $0.15933774511(16)$ \\
$\chi^{2}$ (dof) & $=$ & $46.1(256)$ \\
\hline
\end{tabular}

